\title{
REAL-TIME DYNAMIC MR IMAGE RECONSTRUCTION USING KALMAN FILTERED COMPRESSED SENSING
}

\author{
Chenlu Qiu, Wei Lu and Namrata Vaswani \\ Dept. of Electrical and Computer Engineering, Iowa State University, Ames, IA, \\ $\{$ chenlu, luwei, namrata\}@iastate.edu
}

\begin{abstract}
In recent work, Kalman Filtered Compressed Sensing (KF-CS) was proposed to causally reconstruct time sequences of sparse signals, from a limited number of "incoherent" measurements. In this work, we develop the KF-CS idea for causal reconstruction of medical image sequences from MR data. This is the first real application of KF-CS and is considerably more difficult than simulation data for a number of reasons, for example, the measurement matrix for MR is not as "incoherent" and the images are only compressible (not sparse). Greatly improved reconstruction results (as compared to CS and its recent modifications) on reconstructing cardiac and brain image sequences from dynamic MR data are shown.

Index Terms/Keywords: Compressed Sensing, Kalman Filtered Compressed Sensing, dynamic MRI
\end{abstract}

\section{INTRODUCTION}

In recent work [1], the problem of causally reconstructing time sequences of spatially sparse signals, with unknown and slow timevarying sparsity patterns, from a limited number of linear "incoherent" measurements was studied and a solution called Kalman Filtered Compressed Sensing (KF-CS) was proposed. An important example of this type of problems is real-time medical image sequence reconstruction using MRI, for e.g. dynamic MRI to image the beating heart or functional MRI to image the brain's neuronal responses to changing stimuli(see Fig.1). In these examples, the signal (heart or brain image) is approximately sparse (compressible) in the wavelet transform domain [2],[3]. MRI measures the 2D Fourier transform of the image which is known to be "incoherent" w.r.t. the wavelet basis [2]. Because MR data acquisition is sequential, the scan time (time to get enough data to accurately reconstruct one frame) is reduced if fewer measurements are needed for accurate reconstruction and hence there has been a lot of interest in the MRI community to use compressed sensing (CS) to do this [2],[3].

This idea is first demonstrated in [2] for a single MR image or volume. The work of [3] extended the idea to offline dynamic MRI reconstruction, i.e. it used the entire time sequence of measurements to jointly estimate the entire image sequence (treated it as a 3D x-y$t$ signal, sparse in wavelet domain along the $x-y$ axis and sparse in the Fourier domain along the time axis). But this is a batch solution (needs all measurements first) and also the resulting joint optimization is computationally complex. On the other hand, the solution of [1] is causal and also much faster, and thus can be used to make dynamic MRI real-time. Reduced scan-time and real-time reconstruction are the currently missing abilities that prevent the use of MRI in interventional radiology applications, such as MR-guided surgery[4]. Some other recent work that also targets causal reconstruction of sparse image sequences is [5] and [6].

In this work we use [1] to develop a KF-CS algorithm to causally reconstruct image sequences using MR data. There are some key

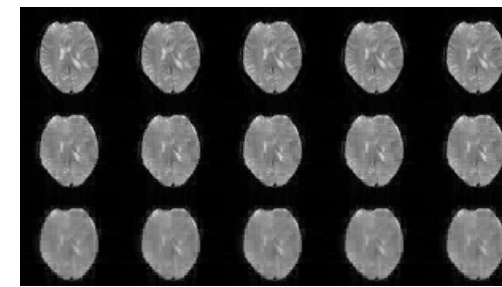

(a) A brain image sequence

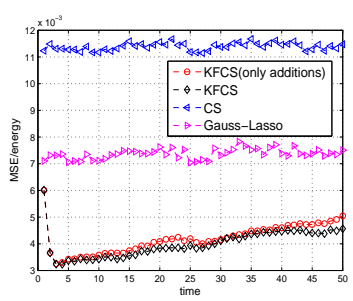

(b) MSE plot
Fig. 1. The three rows of Fig.1(a) show some frames of a brain image sequence(row 1), its MRI reconstructions using KF-CS(row 2), and using CS(row 3). Fig.1(b) is the corresponding MSE plot. $n=2049, m=4096$ and $\sigma_{\text {obs }}^{2}=100$.

differences in our current problem from the simplistic model used in [1] and these require some practical modifications to the algorithm of KF-CS(described in section 2). Additionally, in this work, (i) we develop a method for estimating the prior model parameters from training data(described in section 3.1) and (ii) we use the results of [7] to develop a method for selecting the number of observations required and the parameters used by the CS step of KF-CS(described in section 3.2). Results on reconstructing a cardiac sequence and a brain sequence are discussed in section 4 and they show greatly reduced mean squared error(MSE) when compared to performing $\mathrm{CS}$ at each time as in [2], as well as to some other modifications of CS. For e.g. in Fig 1b, the CS error is more twice that of KF-CS.

\subsection{Problem Formulation}

Let $\left(Z_{t}\right)_{m_{1} \times m_{2}}$ denote the image at time $t$ and let $m:=m_{1} m_{2}$ be its dimension. Let $X_{t}$ denote the $2 \mathrm{D}$ discrete wavelet transform (DWT) of $Z_{t}$, i.e. $X_{t}:=W Z_{t} W^{\prime}$. Let $F$ denote the discrete Fourier transform (DFT) matrix and $Y_{f u l l, t}=F Z_{t} F^{\prime}=$ $F W^{\prime} X_{t} W^{\prime} F^{\prime}$ denote the 2D-DFT of $Z_{t}$. All of this can be transformed to a $1 \mathrm{D}$ problem by using Kronecker product denoted by $\otimes$. Let $y_{f u l l, t}:=\operatorname{vec}\left(Y_{f u l l, t}\right)$ and $x_{t}:=\operatorname{vec}\left(X_{t}\right)$. Then $y_{f u l l, t}=$ $F_{1 D} W_{1 D} x_{t}$ where $F_{1 D}=F \otimes F$ and $W_{1 D}=W \otimes W$. Here, $\operatorname{vec}\left(X_{t}\right)$ denotes the vectorization of the matrix $X_{t}$ formed by stacking the columns of $X_{t}$ into a single column vector. In MR imaging, we capture a set of $n,(n<m)$, Fourier coefficients corrupted by white noise. This can be modeled by applying a $n \times m$ mask, $M$ (which contains a single 1 at a different location in each row and all other entries are zero) to $y_{f u l l, t}$ followed by adding Gaussian noise.

The above can be rewritten using the notation of [1] as

$$
y_{t}=A x_{t}+w_{t}, A:=H \Phi, H:=M F_{1 D}, \Phi:=W_{1 D}
$$

with $w_{t} \sim \mathcal{N}\left(0, \sigma_{o b s}^{2}\right)$ is i.i.d. Gaussian measurement noise. Let $N_{t}$ denote the current set of nonzero coefficients (or significantly 


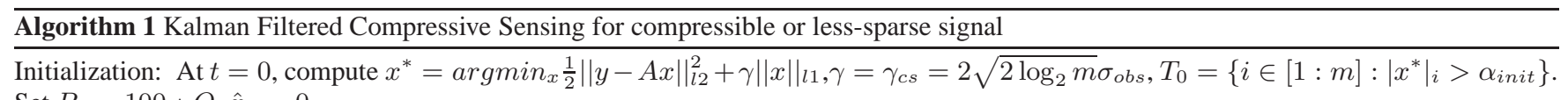
Set $P_{0}=100 * Q, \hat{x}_{0}=0$

For $t>0$, do

1. Temporary $\mathrm{KF}$ using $T \leftarrow T_{t-1}$ :

$$
\begin{aligned}
\left(P_{t \mid t-1}\right)_{T, T} & =\left(P_{t-1}\right)_{T, T}+(Q)_{T, T} \\
K_{t, t m p, T} & =\left(\sigma_{o b s}^{2}\left(P_{t \mid t-1}\right)_{T, T}^{-1}+A_{T}^{\prime} A_{T}\right)^{-1} A_{T}^{\prime} \\
\left(\hat{x}_{t, t m p}\right)_{T} & =\left(\hat{x}_{t-1}\right)_{T}+K_{t, t m p, T}\left(y_{t}-A_{T}\left(\hat{x}_{t-1}\right)_{T}\right),\left(\hat{x}_{t, t m p}\right)_{T^{c}}=\mathbf{0}
\end{aligned}
$$

\section{Run CS on KF error to update nonzero set(Addition/Deletion)}

(a) Compute the filtering error, $\tilde{y}_{t, f}=y_{t}-A_{T} \hat{x}_{t, t m p}$, run CS on $\tilde{y}_{t, f}$, i.e. estimate $\hat{\beta}=\operatorname{argmin}_{\beta} \frac{1}{2}\left\|y_{t, f}-A \beta\right\|_{l 2}^{2}+\gamma\|\beta\|_{l 1}$ with $\gamma=\gamma_{E R C}$. Set $\hat{x}_{t, C S F E}=\hat{x}_{t, t m p}+\hat{\beta}$.

(b) Addition/Deletion: estimate the nonzero set at $t$ as: $T_{c}=\left\{i \in[1: m]:\left|\hat{x}_{t, C S F E}\right|_{i}>\alpha_{a d d}\right\}, T_{t} \leftarrow T_{c}$.

3. Run KF on the current nonzero set $T_{c}$ :

$$
\begin{aligned}
\left(\hat{x}_{t \mid t-1}\right)_{T_{c}} & =\left(\begin{array}{c}
\left(\hat{x}_{t-1}\right)_{T \cap T_{c}, T \cap T_{c}} \\
\mathbf{0}_{T_{c} \backslash T, T_{c} \backslash T}
\end{array}\right),\left(\hat{x}_{t \mid t-1}\right)_{T_{c}^{c}}=\mathbf{0} \\
\left(P_{t \mid t-1}\right)_{T_{c}, T_{c}} & =\left[\begin{array}{c}
\left(P_{t-1 \mid t-1}\right)_{T \cap T_{c}, T \cap T_{c}} \\
\left(P_{0}\right)_{T_{c} \backslash T, T_{c} \backslash T}
\end{array}\right]+(Q)_{T_{c}, T_{c}} \\
K_{t, T} & =\left(\sigma_{o b s}^{2}\left(P_{t \mid t-1}\right)_{T_{c}, T_{c}}^{-1}+A_{T_{c}}^{\prime} A_{T_{c}}\right)^{-1} A_{T_{c}}^{\prime} \\
\left(P_{t}\right)_{T_{c}, T_{c}} & =\left(I-K_{t, T} A_{T_{c}}\right)\left(P_{t \mid t-1}\right)_{T_{c}, T_{c}} \\
\left(\hat{x}_{t \mid t}\right)_{T_{c}} & =\left(\hat{x}_{t \mid t-1}\right)_{T_{c}}+K_{t, T}\left[y_{t}-A\left(\hat{x}_{t \mid t-1}\right)_{T_{c}}\right],\left(\hat{x}_{t \mid t}\right)_{T_{c}}^{c}=\mathbf{0}
\end{aligned}
$$

4. Output $T_{t}, \hat{x}_{t}$ and $\hat{x}_{t, C S F E}$. Compute signal estimation $\hat{z}_{t}=W_{1 D} \hat{x}_{t}$ or $\hat{z}_{t, C S F E}=W_{1 D} \hat{x}_{t, C S F E}$.

nonzero coefficients in case of compressible sequences). For $\left(x_{t}\right)_{N_{t}}$, we assume a spatially independent (but not identically distributed) Gaussian random walk model, i.e.

$$
\begin{aligned}
x_{t} & =x_{t-1}+\nu_{t}, \nu_{t} \sim \mathcal{N}\left(0, Q_{t}\right), \\
\left(Q_{t}\right)_{N_{t}, N_{t}} & =(Q)_{N_{t}, N_{t}} \\
\left(Q_{t}\right)_{N_{t}^{c},[1: m]} & =0, \quad\left(Q_{t}\right)_{[1: m], N_{t}^{c}}=0
\end{aligned}
$$

where $Q$ is a diagonal matrix estimated as explained in section 3.1. The diagonal assumption on $Q$ is a valid one because the wavelet transform is well-known to be a decorrelating transform for natural images [8]. The set, $N_{t}$ of (significantly) nonzero elements of $x_{t}$ changes slowly over time, for e.g. for a $32 \times 32$ block of a cardiac image, $\left|N_{t}\right| \approx 130$ and $\left|N_{t} \backslash N_{t-1}\right| \leq 20$.

\section{KF-CS FOR REAL-TIME DYNAMIC MRI}

The overall idea of Kalman Filtered Compressed Sensing (KF-CS) for a sparse signal sequence is as follows [2]. Let $T_{t}$ denote the KFCS estimated set of (significantly) nonzero coefficients at time $t$, i.e. $T_{t}=\hat{N}_{t}$. At $t=0$, we perform CS on $y_{0}$ followed by thresholding, to estimate $T_{0}$. At any $t$, we first run a reduced order KF for the elements of $T_{t-1}$. Denote its output by $\hat{x}_{t, t m p}$. We use this to compute the filtering error in the observation, $\tilde{y}_{t, f}:=y_{t}-A \hat{x}_{t, t m p}$. If $\tilde{y}_{t, f}$ is larger than usual (its weighted norm is greater than a threshold), it indicates that more coefficients have become nonzero. At this time, we perform CS on $\tilde{y}_{t, f}$ followed by thresholding the output to find new additions to $T_{t-1}$. This is followed by a KF prediction and update step for the current set of nonzero coefficients, $T_{t}$. Coefficients that got falsely added in the past or that became zero over time are removed from $T_{t}$ by thresholding the KF output $\hat{x}_{t}$.

Let $\Delta_{t}:=N_{t} \backslash T_{t-1}$. The reason why KF-CS for sparse signals outperforms CS is that CS uses $y_{t}=A x_{t}+w_{t}$ to estimate the $\left|T_{t-1} \cup \Delta_{t}\right|$-sparse signal, $x_{t}$, while KF-CS uses $\tilde{y}_{t, f}=y_{t}-A \hat{x}_{t}=$ $A \beta_{t}+w_{t}$ to estimate $\beta_{t} \triangleq x_{t}-\hat{x}_{t, t m p}$. As explained in [1], $\beta_{t}=$ $\left[\left(x_{t}-\hat{x}_{t}\right)_{T_{t-1}},\left(x_{t}\right)_{\Delta_{t}}, 0_{\left(T_{t-1} \cup \Delta_{t}\right)}\right]$ is also $\left|T_{t-1} \cup \Delta_{t}\right|$-sparse but is only $\left|\Delta_{t}\right|$-compressible (assuming $\left(x_{t}-\hat{x}_{t, t m p}\right)_{T_{t-1}}$ is small).

Key Differences. KF-CS was designed for estimating a highly sparse signal sequence, with slowly changing nonzero elements' set, from a small number of random Gaussian projections corrupted in Gaussian noise. All nonzero coefficients at a given time were assumed to follow a random walk with equal variance. In the MRI reconstruction problem, there are a few key differences.

1. The matrix, $A=H \Phi$ is no longer random Gaussian. $\Phi$ is the DWT matrix and $H$ contains randomly selected rows from the DFT matrix. The resulting $A$ matrix is not as incoherent (incoherence can be quantified by $\mu=\max _{i \neq j}\left|A_{i}^{\prime} A_{j}\right|$ ) as a random Gaussian matrix of the same size.

2. The wavelet transform coefficients' vector, $x_{t}$, of a real medical image (e.g. cardiac or brain) is only compressible (not sparse) and the number, $S_{t}$, of "significantly" nonzero coefficients, (as a percentage of the signal size, $m$ ) is much larger than what was used in the simulations in [1].

3. Different wavelet coefficients have different variances, and in fact the nonzero coefficients that get added/deleted over time are typically the smaller variance ones.

4. The problem dimension, $m$ is much larger (e.g. $m=4096$ ). 
Modifications. We describe below our modifications to address the above issues. The final algorithm is summarized in Algorithm 1.

Use of Lasso. Because of 1 and 2, the Dantzig selector, which was designed for very sparse signals, estimated from highly incoherent observations does not work well. We replace it by the Lasso (see step 2a of Algorithm 1), which was also used in [2].

Dealing with false additions and misses. Even with Lasso, because of 3 , either many of the (significantly) nonzero coefficients never get added to $T_{t}$, i.e. $T_{t} \backslash N_{t}$ is large or there are too many false additions, $\Delta=T_{t} \backslash N_{t}$. In the latter case, the increase in $\left|T_{t}\right|$ may result in a singular $A_{T_{t}}^{\prime} A_{T_{t}}$ (making the KF unobservable). Because of 1 , this begins to occur for smaller values of $\left|T_{t}\right| / n$ than if $A$ were random Gaussian (and of course occurs more often when $n$ is smaller, e.g. when $n=308$ for a $32 \times 32$ cardiac image sequence).

To prevent this, the addition/deletion threshold, $\alpha_{a d d}$, needs to be large, but this results in larger $\Delta=N_{t} \backslash T_{t}$. Note that the estimation error along $T=T_{t},\left(x_{t}-\hat{x}_{t}\right)_{T}$, depends linearly on $A_{T}^{\prime} A_{\Delta}$. Because of larger sized $\Delta$ and because of $1, A_{T}^{\prime} A_{\Delta}$ is no longer very small and thus $\left(x_{t}-\hat{x}_{t}\right)_{T}$ is also not very small. By using the output of the CS step, $\hat{x}_{t, C S F E}=\hat{x}_{t, t m p}+\hat{\beta}_{t}$, as the final output (instead of using the KF output, $\hat{x}_{t}$ ), we ensure that at least the CS estimate of $\left(\beta_{t}\right)_{T}$ is included in the final estimate. For the same reason, we also use the $\hat{x}_{t, C S F E}$ for deletion, in fact we combine addition and deletion into a single step (see step $2 \mathrm{~b}$ of Algorithm 1).

Initial covariance. Since there is an unknown delay in detecting new additions to the nonzero coefficients' set, the initial error covariance of newly added coefficients is never correctly known. We use an arbitrarily large value, $P_{0}=100 Q$ for it (the estimate for the new coefficients will thus be closer to an LS solution).

Efficient Implementation. When $m$ is large, a direct implementation of KF-CS becomes very slow. We make it faster using some simple reformulations such as solving a $2 \mathrm{D}$ version of lasso and using the algorithm of [2] or using standard matrix algebra tricks to speed up matrix multiplications and inversions in the KF step.

\section{PARAMETER ESTIMATION}

Section 3.1 discuss how we estimate $Q$ and section 3.2 discuss how we select $n$ and $\gamma$ using ERC.

\subsection{Estimating $Q$}

We consider two models for $Q$. The first one assumes $Q$ to be a diagonal matrix with different entries while the second assumes $Q$ is diagonal with all equal entries. The second model will have more bias (since finer scale wavelet coefficients always have smaller variance than coarser scale ones) but will have smaller variance and hence is a better idea when limited training data is available. We can compute an approximate Maximum Likelihood (ML) estimate of $Q$ under either model: we pick a zeroing threshold, set all coefficients below it to zero and use the rest of the coefficients to compute the ML estimate. The algorithm is summarized in Algorithm 2. $\left|\delta_{i}\right|$ in step 2 stands for the times of nonzero occurrence for $i^{\text {th }}$ entry of $x_{t}$ and $\left|\delta_{i}\right|=0$ implies the $i t h$ entry, $x_{t, i}$, is zero or equal at all time.

\subsection{Selecting $n$ and $\gamma$ using ERC}

The lasso estimator is defined as the solution to

$$
\arg \min _{x_{t}} \frac{1}{2}\left\|y_{t}-A x_{t}\right\|_{l 2}^{2}+\gamma\left\|x_{t}\right\|_{l 1}
$$

where $\gamma$ is a regularization parameter that determines the tradeoff between the data consistency and the sparsity. We use Theorem 8 of [7] to develop an algorithm to select $\gamma$ and $n$ using training data. Let $\Lambda$ be the nonzero set of $x_{t}$ which we want to estimate. The exact $\frac{\overline{\text { Algorithm } 2 Q \text { estimation }}}{\text { 1. Zero out "compressible"(nearly zero) coefficients }}$

(a) Select zeroing threshold

- For $t=1$ : $L_{\text {train }}$, here, $L_{\text {train }}$ denotes the length of training sequence,

- Compute $x_{t}=W_{1 D}^{\prime} z_{t}$. Arrange $\left|x_{t, i}\right|$ in decreasing order of magnitude, i.e. $\left|x_{t, 1}\right|>$ $\left|x_{t, 2}\right|>\cdots>\left|x_{t, m}\right|$.

- Compute the smallest $S_{t}$ such that $\Sigma_{j \leq S_{t}}\left|x_{t, i}\right|^{2}>99.9 \% x_{t}^{\prime} x_{t}$, set threshold $\alpha_{t}=\left|x_{t, S_{t}}\right|$.

- Average $\alpha_{t}$ over $t, \alpha=\frac{\Sigma_{t} \alpha_{t}}{L_{\text {train }}}$.

(b) Zero out "compressible" coefficients

- For $t=1: L_{\text {train }}$, if $\left|x_{t, i}\right|<\alpha$, set $x_{t, i}=0$. Set $N_{t}=\left\{i \in[1: m]:\left|x_{t, i}\right| \geq \alpha\right\}$.

2. Estimate $Q$ using nonzero coefficients. Compute $\delta_{i}:=\{t$ : $\left.x_{t, i}-x_{t-1, i} \neq 0\right\}$

- For $Q$ with different diagonal values,

$$
\begin{aligned}
\text { - } & \text { if }\left|\delta_{i}\right| \geq 1,\left(\sigma_{\text {sys }}^{2}\right)_{i}=\frac{1}{\left|\delta_{i}\right|} \Sigma_{t=2}^{L_{t \operatorname{train}}}\left(x_{t, i}-\right. \\
& \left.x_{t-1, i}\right)^{2} . \\
\text { - } & \text { if }\left|\delta_{i}\right|=0,\left(\sigma_{\text {sys }}^{2}\right)_{i}=0.9 \min _{\left\{j:\left|\delta_{j}\right| \geq 0\right\}} \sigma_{\text {sys }, i}^{2} \\
\text { - } & \text { Set } Q_{\text {diff }}=\operatorname{diag}\left(\left(\sigma_{\text {sys }}^{2}\right)_{i}\right) .
\end{aligned}
$$

- For $Q$ with equal diagonal values,

$$
\begin{aligned}
& - \text { Compute } \quad\left(\sigma_{\text {sys }}^{2}\right)_{i} \\
& \frac{1}{\Sigma_{j}\left(\left|\delta_{j}\right|\right)} \Sigma_{j \in\left\{j\left\|\delta_{j}\right\| \geq 1\right\}} \Sigma_{t=2}^{L_{\text {train }}}\left(x_{t, i}-x_{t-1, i}\right)^{2} . \\
& \text { Set } Q_{\text {same }}=\operatorname{diag}\left(\left(\sigma_{\text {sys }}^{2}\right)_{i}\right) .
\end{aligned}
$$

recovery coefficient, $\operatorname{ERC}(\Lambda):=1-\left\|A_{\Lambda}^{\dagger} A_{\Lambda^{c}}\right\|_{l 1}>0$ is one of the conditions for Theorem 8 to hold. Here, $\dagger$ denotes pseudoinverse.

Selecting $n$ : Given $y_{t}$ and the ground truth data $x_{t}$. Threshold $x_{t}$ to define its nonzero set $N_{t}$. Find the smallest value of $n$ such that $E R C\left(N_{t} \backslash N_{t-1}\right)>0$ for at least $90 \%$ of times.

Selecting $\gamma$ : Theorem 8 of [7] guarantees that if $\gamma$ is large enough (its correlation condition is satisfied), the lasso estimator will have no falsely nonzero coefficients, but may end up not adding the small coefficients. For sufficiently sparse signal sequences, one can use the correlation condition given in this result to find the minimum value of $\gamma$ required for a given training sequence. This can be done

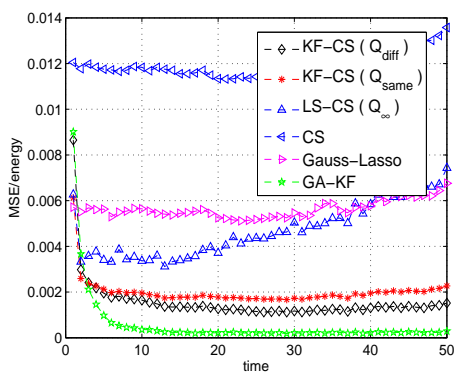

Fig. 2. Comparison of KF-CS with different model of $Q$. $n=$ $308, m=1024$ and $\sigma_{o b s}^{2}=25 . Q_{\infty}$ denotes $Q=\infty$. 


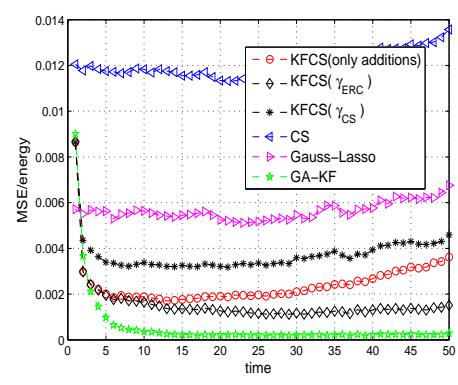

(a) $n=308, m=1024, \sigma_{\text {obs }}^{2}=25$

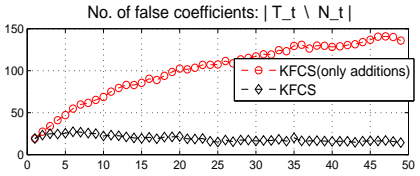

No. of missing coefficients: $\left|N \_t \backslash T \_t\right|$

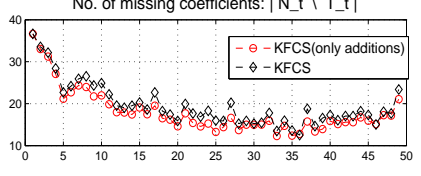

(b) $n=308, m=1024, \sigma_{\text {obs }}^{2}=25$

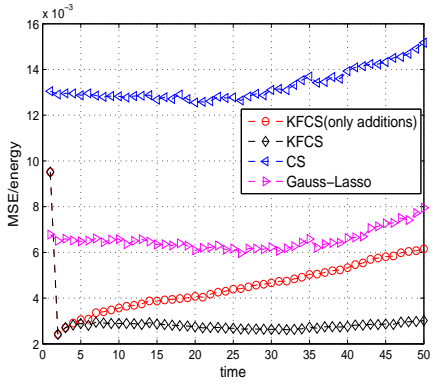

(c) $n=512, m=1024, \sigma_{\text {obs }}^{2}=25$

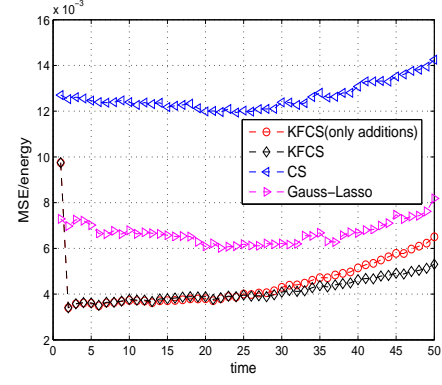

(d) $n=308, m=1024, \sigma_{\text {obs }}^{2}=25$

Fig. 3. Fig3(a) is MSE/energy plot for sparsified cardiac image sequence using $\gamma$ obtained from ERC-based method and $\gamma=\gamma_{c s}$. Fig3(b)

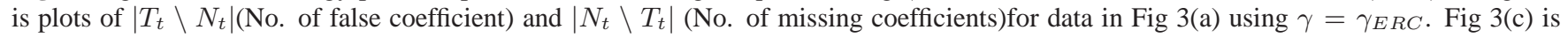
MSE/energy plot for true cardiac image sequence with $\gamma=2<\gamma_{E R C}$. Fig 3(d) is MSE/energy plot for true cardiac image sequence with $\gamma=0.005<<\gamma_{E R C}$

as follows. Starting with $T_{0}=N_{0}$, do the following for $t=1$ to $L_{\text {train: }}$ (a) compute $\Delta_{t}=N_{t} \backslash T_{t-1}$; (b) compute $\operatorname{ERC}\left(\Delta_{t}\right)$; and (c) run step 2 of Algorithm 1 with $\gamma=\gamma_{t}=\frac{\left\|\tilde{y}_{t, f}-A_{\Delta_{t}} A_{\Delta_{t}}^{\dagger} \tilde{y}_{t, f}\right\|}{E R C\left(\Delta_{t}\right)}$. Use $\gamma_{E R C}=\max _{t} \gamma_{t}$ for test data.

In practice, for compressible data, using $\gamma=\gamma_{E R C}$ adds too few coefficients. In this case we compute $\gamma_{E R C}$ using a sparsified version of the true training sequence, but use a smaller value of $\gamma$ for the test data. This allows more coefficient additions.

\section{EXPERIMENT RESULTS}

We evaluated our algorithm on cardiac and a brain image sequences. Fig. 1 is the comparison of KF-CS reconstructed image sequence with that of CS for brain data. Notice the white region in the center is much more blurred in CS reconstruction than in KFCS. For the results of Fig 2 and 3, we first selected a $32 \times 32$ region in the image, used this sequence as the test data. MRI was simulated by taking the 2D-DFT of the given image sequence, selecting a random set of $n$ Fourier coefficients using the variable-density undersampling scheme proposed in [2] and adding i.i.d. Gaussian noise to them. In the plots of Fig 2, 3, we plot MSE/energy := $=\mid x_{t}-\hat{x}_{t}\left\|_{l 2}^{2} /\right\| x_{t} \|_{l 2}^{2}$ which was computed by averaging over 50 Monte Carlo simulations for cardiac sequence. Fig. 2 compares KF-CS using $Q_{\text {diff }}, Q_{\text {same }}$ and using $Q=\infty$ (replacing KF by LS). $n=308$ observations were taken while $\left|N_{t}\right| \approx 130$ and $\left|\Delta_{t}\right| \leq 20$. Since $\sigma_{\text {obs }}^{2}$ is large, KF-CS which makes use of prior model knowledge performs much better than LS-CS. Also, KF-CS with $Q_{\text {diff }}$ outperforms KFCS with Qsame.

In Fig3(a), we show that KF-CS with $\gamma_{E R C}$ outperforms KFCS with an arbitrary (large) choice of $\gamma$, KF-CS (only additions), Gauss-Lasso and CS. The comparison is done for sparsified cardiac sequence. KF-CS(only additions) refers to algorithm proposed in [1], in which we kept adding new additions to the nonzero coefficients set at each $t$ without dealing with false additions. For GaussLasso, we first estimated nonzero set by Lasso, followed by LS on it (similar to Gauss-Dantzig[9]). Genie-aided KF (GA-KF)is a KF that knows $N_{t}$ at each $t$. This serves as a MSE lower bound. Fig 3(b) compares number of false coefficients and number of missing coefficients for KF-CS and KF-CS (only additions). Fig3(c) and Fig3(d) compare KF-CS with KF-CS(only additions), CS and Gauss-Lasso for true(not sparsified) cardiac data. In Fig3(c), we set $\gamma=2<\gamma_{E R C}$ with $n=512$ and $m=1024$. In Fig3(d), we set $\gamma=0.005<<\gamma_{E R C}$ while $n=308<<512$. When $n$ is very small, we use smaller $\gamma$ and only run KF on "larger" coefficients while use $\hat{x}_{C S F E}$ as final output.

\section{CONCLUSIONS AND FUTURE WORK}

In this paper, we have developed the KF-CS idea for causal reconstruction of medical image sequences from MR data and have shown greatly improved reconstruction results on cardiac dynamic and brain fMRI data, as compared to CS [2] and its modifications. This is the first real application of KF-CS and is considerably more difficult than simulation data because the measurement matrix for MR is not as incoherent as a random Gaussian matrix and because the different wavelet coefficients have vastly different magnitudes and variances. Future work will involve a rigorous analysis of the proposed algorithmic ideas and using it to propose a novel KF-CS based algorithm for compressible sequences.

\section{REFERENCES}

[1] N. Vaswani, "Kalman filtered compressed sensing," in IEEE Intl. Conf. Image Proc. (ICIP), 2008.

[2] M. Lustig, D. Donoho, and J. M. Pauly, "Sparse mri: The application of compressed sensing for rapid mr imaging," Magnetic Resonance in Medicine, vol. 58(6), pp. 1182-1195, December 2007.

[3] U. Gamper, P. Boesiger, and S. Kozerke, "Compressed sensing in dynamic mri," Magnetic Resonance in Medicine, vol. 59(2), pp. 365-373, January 2008.

[4] A. J. Martin, O. M. Weber, D. Saloner, R. Higashida, M. Wilson, M. Saeed, and C.B. Higgins, "Application of MR Technology to Endovascular Interventions in an XMR Suite," Medica Mundi, vol. 46, December 2002.

[5] C. Rozell, D. Johnson, R. Baraniuk, and B. Olshausen, "Locally competitive algorithms for sparse approximation," in IEEE Intl. Conf. Image Proc. (ICIP), 2007.

[6] H. Jung, K. Sung, K. S. Nayak, E. Y. Kim, and J. C. Ye, "kt FOCUSS: a general compressed sensing framework for high resolution dynamic MRI," Magnetic Resonance in Medicine, vol. 35, No. 6, 2313-2351, Dec.2007.

[7] Joel A.Tropp, "Just Relax: Convex Programming Methods for Identifying Sparse Signals in Noise," IEEE Trans. on Info. Th., vol. 52, no. 3, March. 2006.

[8] Anil K. Jain, Fundamentals of Digital Image Processing, Prentice-Hall of India, 1995.

[9] E. Candes and T. Tao, "The dantzig selector: statistical estimation when p is much larger than n," Annals of Statistics, 2006. 